\title{
Structural Similarities and Differences Between Neurofilament Proteins from Five Different Species as Revealed Using Monoclonal Antibodies
}

\author{
Virginia M.-Y. Lee, Martin J. Carden, and William W. Schlaepfer \\ The Division of Neuropathology, Department of Pathology and Laboratory Medicine, University of Pennsylvania \\ School of Medicine, Philadelphia, Pennsylvania 19104-6079
}

Neurofilament (NF) proteins isolated from human, rabbit, rat, and chicken spinal cord white matter were immunoblotted with monoclonal antibodies (MAbs) raised to bovine NF immunogens. The aim of these experiments was to test the degree of epitope conservation among NF proteins from different vertebrate species. In so doing, the validity of using antibodies raised to NF from one species (cow) for detecting NF proteins of other species was also tested. The MAbs used for these experiments were characterized using bovine NFs to define polypeptide specificity, the approximate location of epitopes within each NF polypeptide, and the effects on antibody recognition of the presence or absence of phosphorylated residues in these polypeptides (see Carden et al., 1985). Our findings indicate that epitopes located in the alpha-helical core domains of NF-H, NF-M, and NF-L are distinct from each other, yet are strongly conserved among the different species. Epitopes located in the noncore or peripheral domains of the NF polypeptides show variable degrees of cross-species preservation. For example, all epitopes in the peripheral domains of bovine NF-H that require the presence of phosphate groups for recognition (phosphorylation dependent) are widely expressed in all species studied. Many phosphorylation-dependent epitopes of bovine NF-H are also present in bovine NF-M, as well as in NF-H of non-bovine species. In addition, epitopes in the peripheral domain of NF-H that require dephosphorylation of NF polypeptides for recognition (dephosphorylation dependent) are also found in $\mathbf{N F}-\mathbf{H}$ of other mammalian species but not in NF-H of chicken. On the other hand, other epitopes in the noncore domains of NF-M and NF-L show limited, if any, cross-species reactivity. For example, phosphorylation-independent epitopes on bovine NF-M are not present in any other species studied. Moreover, those MAbs recognizing epitopes in noncore domains of bovine NF-L show no clear pattern of cross-species reactivity. Some are negative in all species, while others react with homologous domains from certain species but not from others. In all cases, NF-L epitopes are unmodified after phosphatase treatment.

The results presented here suggest that certain domains of NF polypeptides have been conserved while others have undergone structural divergence during evolutionary development. Preservation of core domains among NF polypeptides probably

\footnotetext{
Received Sept. 6, 1985; revised Nov. 21, 1985; accepted Jan. 30, 1986.

This work was supported by NIH Grants NS-18616 and NS-15572 and by a Postdoctoral Fcllowship from the Amyotrophic Latcral Sclcrosis Foundation of America (M.J.C.). Appreciation is expressed to Connie Page, Beatrice Engelsberg, and Donald Gordon for excellent technical assistance and to Dr. John Trojanowski for advice and discussion.

Correspondence should be addressed to Dr. Virginia M.-Y. Lee, Division of Neuropathology, Department of Pathology and Laboratory Medicine, 449 Johnson Pavilion/G2, University of Pennsylvania School of Medicine, Philadelphia, PA 19104.

Copyright (C) 1986 Society for Neuroscience $0270-6474 / 86 / 082179-08 \$ 02.00 / 0$
}

bears on their functional importance in NF assembly. Extensive cross-species immunoreactivities among phosphorylation-dependent epitopes in the peripheral domains of $\mathrm{NF}-\mathrm{H}$, however, may well reflect the importance of phosphorylation in mediating interactions between NFs and the surrounding cytoskeletal and/ or other elements of neuronal cytoplasm.

Neurofilaments (NFs), the neuron-specific class of intermediate filaments (IFs), are major cytoskeletal components of most neurons. While non-neuronal IF components have $M_{\mathrm{r}} 40-70,000$, NFs are constructed from 3 polypeptides of relatively high $(200,000)$, medium $(150,000)$, and low $(70,000)$ apparent molecular weights, denoted here as NF-H, NF-M, and NF-L, respectively. Despite size differences, however, all IF components, including the 3 NF subunits, contain a core domain $\left(M_{\mathrm{r}} 35-\right.$ $40,000)$ that is rich in alpha-helical configuration and capable of forming double-stranded coiled-coils (Geisler et al., 1985c), believed to be the structural basis for assembly of the filamentous backbone in all IFs (Crewther et al., 1984; Fuchs and Hanukoglu, 1983; Weber and Geisler, 1984). Differences between IF components arise from variations in the $\mathrm{NH}_{2-}$ and $\mathrm{COOH}-$ terminal sequences flanking their structurally preserved cores. In particular, the large size of NF-H and NF-M arises from marked elongation of $\mathrm{COOH}$-terminal domains (Geisler et al., 1983), termed peripheral segments here, since they can be cleaved from NF without disrupting filament integrity (Chin et al., 1983). Indeed, chymotrypsin releases soluble fragments with apparent $M_{\text {r }} 160,000$ and 120,000 (Chin et al., 1983; Julien and Mushynski, 1983) that represent almost the complete COOH-terminal regions of NF-H and NF-M, respectively (Geisler et al., 1983). NF-M and, especially, NF-H are heavily phosphorylated (e.g., Jones and Williams, 1982) at sites located within their peripheral COOH-terminal domains (Geisler et al., 1985a; Julien and Mushynski, 1983). It is likely that NF-M and NF-H peripheral domains are located on the external aspect of the filament, if not in the cross-bridges that connect NF in situ (Hirokawa et al., 1984), and hence mcdiate functional interactions between NF and surrounding cytoplasmic structures. Figure 1 summarizes the above information about NF polypeptide structure, enzymatic cleavage, and phosphorylation.

Different regions of NF polypeptides have been mapped by immunological methods. For example, the monoclonal antibody (MAb) designated anti-IFA (Pruss et al., 1981) recognizes an epitope present in the core domains of all 3 NF proteins, as well as in those of most other IF components (Geisler et al., 1983). Other MAbs are specific for NF but cross-react with 2 or more subunits (e.g., Calvert and Anderton, 1982; Lee et al., 1982; Shaw et al., 1984), especially when the epitopes reside in the phosphorylated peripheral domains of NF-H and NF-M (Carden et al., 1985; Sternberger and Sternberger, 1983). In some cases, the same epitopes, possibly phosphorylation de- 


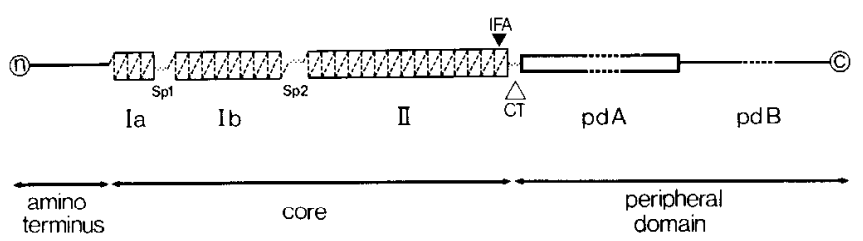

Figure 1. Schematic diagram of NF polypeptide structure. General structure of intermediate filament polypeptides, including NF-H, NF-M, and NF-L, consists of a central core domain $\left(M_{\mathrm{r}} 35-40,000\right)$ flanked by a rclatively small $\mathrm{NH}_{2}$-terminal domain $\left(M_{\mathrm{r}} 10,000\right)$ and a variably sized COOH-terminal domain $\left(M_{r} 15-160,000\right)$. The core domain contains several alpha-helical-rich segments (Ia, Ib, and II) separated by spacer elements ( $S p 1$ and $S p 2)$, as well as the epitope site $(I F A)$ of the Pruss antibody (Pruss et al., 1981). The COOH-terminal domains (peripheral domains) of NF-H and NF-M are cleaved from the core domains by mild chymotryptic digestion $(C T)$. Phosphorylation sites are located in regions of the peripheral domains, which are situated internally $(p d A)$, rather than externally $(p d B)$, on the polypeptide (after Geisler et al., 1985a-c).

pendent, occur in repetitive sequences along individual subunits (Hogue-Angeletti et al., 1985; Liem et al., 1985). Still other MAbs recognize epitopes that are unchanged by NF dephosphorylation (phosphorylation-independent epitopes), while other sites on peripheral domains are exposed only in nonphosphorylated and/or enzymatically dephosphorylated NF (Bennett and DiLullo, 1985; Carden et al., 1985; Lee et al., 1986; Sternberger and Sternberger, 1983). The extent to which such biochemically defined anti-NF MAbs retain immunoreactivity to subunit proteins of different vertebrate species has received only limited attention (Shaw et al., 1984). This information has practical value in assessing the utility of immunological probes when applied to heterologous species. In addition, it provides insights about structural and functional aspects of NF by defining regions of subunits that have been conserved during evolution and those in which mutations can be tolerated.

\section{Materials and Methods}

\section{Nervous tissues}

Rat and rabbit spinal cords were processed immediately after sacrifice. Bovine and chicken cords were obtained from a local slaughterhouse within 10 min post mortem, transported on ice to the laboratory, and processed within $2 \mathrm{hr}$. Human spinal cord was processed within an 8 hr post-mortem interval. Bovine cords were enriched for white matter by splitting them longitudinally along their anterior median fissure and scraping away most of the gray matter from the inner surface using a blunt spatula.

\section{Isolation of NF and immunoblotting}

All isolation procedures were performed at $4^{\circ} \mathrm{C}$. Bovine NFs were isolated exactly as previously described (Carden et al., 1985). Spinal cord tissues from human, rat, rabbit, or chicken were minced and homogenized in Dounce homogenizers using $10 \mathrm{ml}$ of buffer $\mathrm{A} / \mathrm{gm}$ tissue. Buffer A contained $0.1 \%$ Triton X-100, $50 \mathrm{~mm}$ Tris/ $\mathrm{HCl}, 100 \mathrm{~mm} \mathrm{NaCl}, 1$ $\mathrm{mm}$ dithiothreitol, and $2 \mathrm{~mm}$ FGTA, all at $\mathrm{pH}$ 7.0. Homogenates were clarified by centrifugation at low speed $(3000 \times g)$ for $5 \mathrm{~min}$. Floating matter and pellets were rehomogenized and centrifuged twice, again at low speed. The resulting supernatant fractions were combined, and 40 $\mathrm{ml}$ aliquots were layered carefully onto $18 \mathrm{ml}$ pads of sucrose buffer (1.5 M sucrose, $100 \mathrm{~mm} \mathrm{NaCl}, 2 \mathrm{~mm}$ EGTA, $5 \mathrm{~mm}$ dithiothreitol, and $50 \mathrm{~mm}$ Tris/ $\mathbf{H C l}, \mathrm{pH} 7.0$ ). Tubes were centrifuged overnight (approximately $18 \mathrm{hr})$ at high speed $(75,000 \times g)$. Pellets were homogenized in a large excess (at least 50-fold) of buffer $A$ lacking Triton, centrifuged at high speed $(200,000 \times g)$ for $2 \mathrm{hr}$, and immediately resuspended in SDS sample buffer. NF samples over a 10 -fold range of protein concentration were subjected to SDS-PAGE on $7.5 \%$ polyacrylamide gels and immunoblotted exactly as described previously (Carden et al., 1985; Lee et al., 1986; Schlaepfer et al., 1984).
In some experiments, nitrocellulose blots containing normal NF proteins were incubated with phosphatase in order to dephosphorylate the bound protein. The method originated by Sternberger and Sternberger (1983) was used with the modifications of Carden et al. (1985). Chymotryptic fragments of bovine NF proteins were generated and immunoblotted as described previously (Carden et al., 1985). MAbs were graded as negative against NFs from any particular species if no immunostaining of blots could be detected in any of the protein concentrations used.

\section{Monoclonal antibodies}

Lewis rats werre immunized with gel-excised bovine NF proteins, and their splenocytes were fused with mouse myeloma cells (SP2/0-Ag14; Human Genetic Cell Center, Philadelphia) as described before (Lee et al., 1982, 1984, 1985). The MAbs designated here as the Ta, Oc, and Se series were produced from rats immunized with gel-excised NF-H, NF-M, and NF-L, respectively. Enzymatically dephosphorylated NF$\mathrm{H}$ and NF-M, in combination, were used to produce the $\mathrm{dP}$ series of MAbs (Lee et al., 1986). All hybridomas were subcloned. MAbs specific for vimentin and glial fibriallary acid protein (GFAP) were from previous studies (Lee and Page, 1984; Lee et al., 1984).

\section{Results}

Protein composition of $\mathrm{NF}$-rich preparations from different species

Figure 2 shows SDS-PAGE analysis of protein composition in NF-rich extracts obtained from spinal cord tissue of 4 mammalian species: rat, rabbit, human, and cow (lanes 2-5, respectively). Gel mobilities of the 3 major NF polypeptide components, NF-H, NF-M, and NF-L, vary according to species, as is already well established (e.g., Brown et al., 1981; Chiu et al., 1980; Davison and Jones, 1980; Eagles et al., 1980; Liem et al., 1978; Phillips et al., 1983; Shaw et al., 1984), and, in the case of rabbit, NF-H resolves as a doublet (Czosnek et al., 1980; Willard et al., 1976). In addition, there are varying quantities of other cytoskeletal proteins in the $M_{r} 40-60,000$ region, including actin, GFAP, tubulin, and vimentin. Vimentin and GFAP were identified in these preparations by immunoblot (data not shown) using MAbs previously described (Lec and Page, 1984; Lee et al., 1984).

\section{Antibody specificity for bovine NF proteins}

Bovine NF proteins were used to raise and characterize the MAbs for this study. Antibody specificities were determined using an enzyme-linked immunosorbent assay (Lee and Page, 1984) and immunoblotting (e.g., Carden et al., 1985; Trojanowski et al., 1985). NF polypeptide specificity was the first criterion used to divide the anti-NF MAbs into the separate categories listed in Table 1. Representative immunoblot patterns are shown in Figure 3: 13 MAbs recognize only NF-H (e.g., lane 5): 25 are specific for NF-M alone (lane 6); 10 bind only NF-L (lane 9); 87 MAbs bind epitopes shared by NF-H and NF-M (lanes 3 and 4); 4 MAbs recognize both NF-M and NF-L (lanes 7 and 8); and 6 MAbs bind all 3 NF components (lanes 1 and 2). Despite equivalent loadings of NF proteins on the immunoblots in Figure 3, the width of immunoreactive bands varied among MAbs. This might be explained by the heterogeneity of polypeptides migrating at the same $M_{\mathrm{r}}$ position or by a concentration effect in which antibodies with weak affinities might stain only areas where the antigen is most concentrated, i.e., at the center of the protein band. Differences in the intensities of cross-reacting bands (compare lanes 1 and 2 in Fig. 3) show that shared epitopes are similar, but not identical, among NF proteins.

A few MAbs recognize proteins of $M_{\mathrm{r}}$ below 60,000 in addition to 1 or more of the 3 major NF components (e.g., lanes 8 and 10 in Fig. 3). The lower band in lane 10 (Fig. 3) was identified as vimentin using a monospecific MAb to this protein 


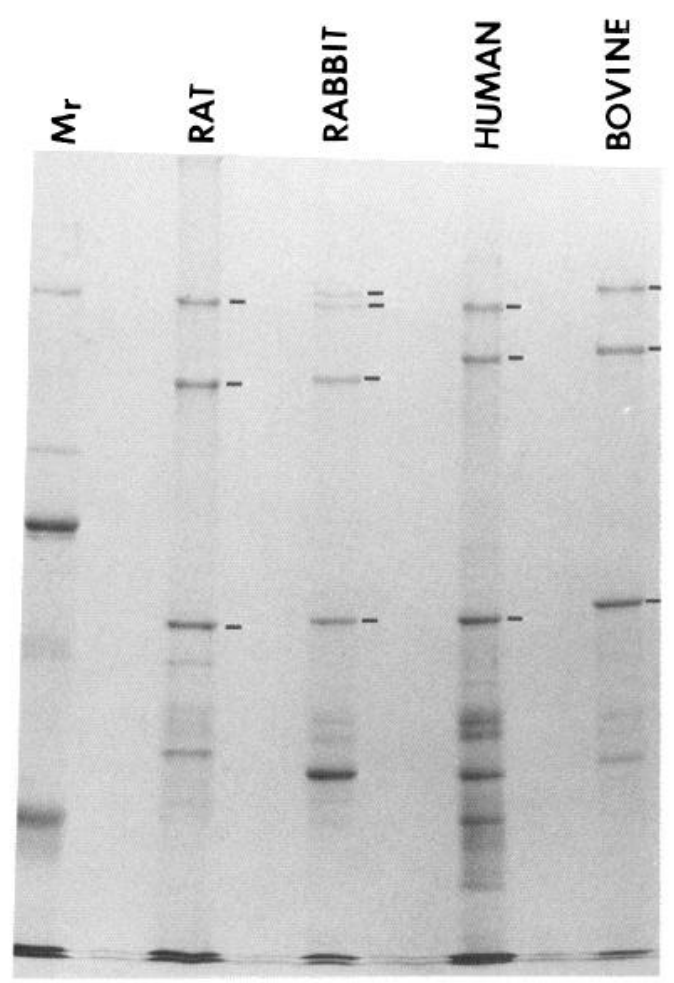

Figure 2. Gel analysis of NF proteins from different species. This $7.5 \%$ polyacrylamide gel contains NF-rich samples extracted from rat (lane 2 ), rabbit (lane 3), human (lane 4), and bovine (lane 5) spinal cords. Marker proteins in lane 1 were myosin $\left(M_{\mathrm{r}} 200,000\right)$, beta-galactosidase $\left(M_{\mathrm{r}} 115,000\right)$, phosphorylase $\mathrm{b}\left(M_{\mathrm{r}} 96,000\right)$, bovine serum albumin $\left(M_{\mathrm{r}}\right.$ $66,000)$, and ovalbumin $\left(M_{\mathrm{r}} 43,000\right)$. The heavy, middle, and light polypeptides of NF are indicated for each sample. Note that rabbit NF-H migrates as a doublet (see also Czosnek et al., 1980; Willard et al., 1980).

(Lee and Page, 1984). However, some of the cross-reacting proteins (e.g., lane 8 in Fig. 3) were neither GFAP nor vimentin.

The second criterion used to distinguish groups of antibodies was the approximate location of epitopes recognized by MAbs within NF polypeptides (Table 1). This was achieved by immunoblotting defined chymotryptic fragments of NF proteins (details are given in Carden et al., 1985). MAbs were defined as binding peripheral epitopes if they recognized the soluble chymotryptic fragments of NF-H and/or NF-M that are released after limited digestion of intact bovine NF (Chin et al., 1983; Julien and Mushynski, 1983). Core-binding MAbs were identified using immunoblots of chymotryptic digests from individual NF polypeptides and depended on the ability of MAbs to recognize the same $M_{\mathrm{r}} 20-45,000$ fragments as recognized by the MAb called anti-IFA (Pruss et al., 1983), which binds a welldefined epitope (Geisler et al., 1983) located in the core regions of each NF component, as shown in Figure 1. MAbs that recognized NF-L, but not the same $40,000 M_{\mathrm{r}}$ fragments as recognized by anti-IFA, were consequently considered to bind noncore epitopes, i.e., $\mathrm{NH}_{2}$ - or $\mathrm{COOH}$-terminal domains. MAbs directed against core epitopes in NF-H and NF-M (categories III and VI) were very rare. MAbs recognizing peripheral domains of NF-H and NF-M were, on the other hand, extremely common: 123 such MAbs were obtained, and the majority (87 MAbs) recognized both NF-H and NF-M (categories IX and $\mathrm{X})$.

The third criterion used to classify MAbs for Table 1 was their ability to detect NF proteins in different states of phosphorylation. The phosphorylation of the 2 largest NF proteins can alter their antigenicity (Bennett and DiLullo, 1985; Carden

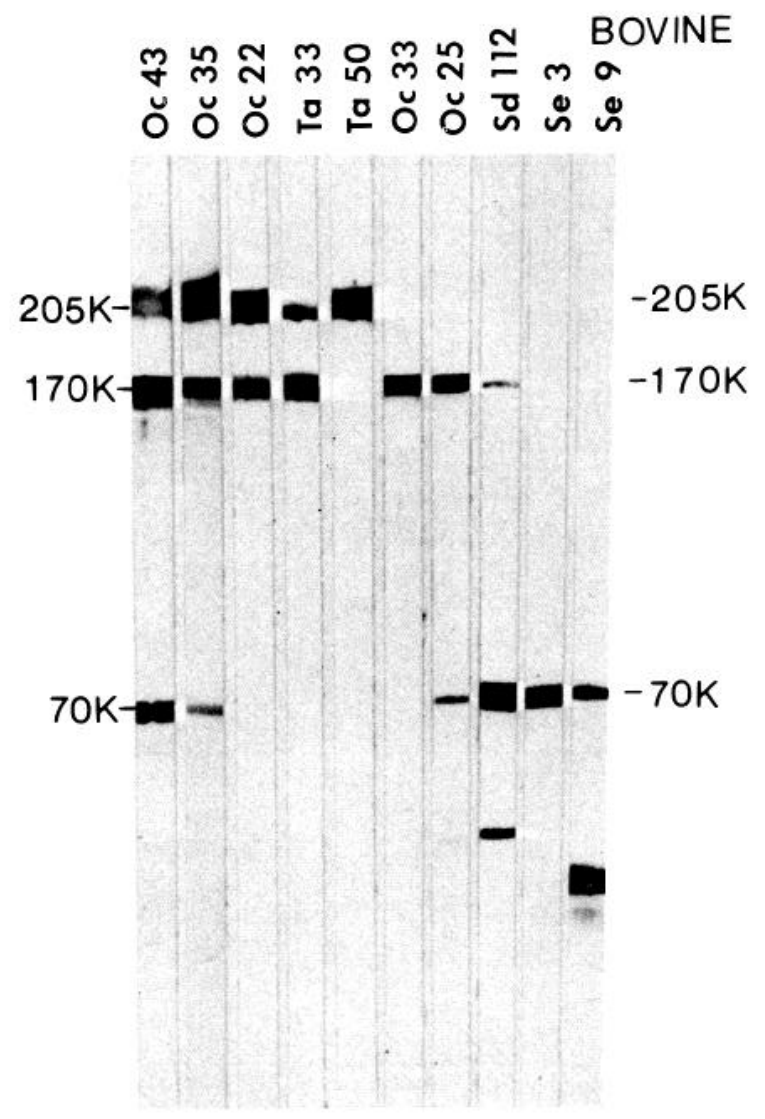

$122 \quad 3 \quad 4 \quad 566788910$

Figure 3. Immunoblots of bovine NF proteins. Bovine NF-rich preparations were separated on a $7.5 \%$ polyacrylamide gel and transferred to nitrocellulose paper. Strips cut from this replica were incubated with MAbs in the form of undiluted hybridoma culture supernatants. The identity of the particular MAb used for each strip is indicated at the top.

et al., 1985; Lee et al., 1986; Sternberger and Sternberger, 1983). Hence, MAbs were used for comparative immunoblotting of freshly isolated (predominantly phosphorylated) and enzymatically dephosphorylated NF proteins. These studies revealed 3 kinds of MAb: (1) those recognizing NF polypeptides equally well before and after phosphatase treatment, termed phosphorylation-independent, or $P($ ind $)$ MAbs; (2) those having higher affinity for phosphorylated antigens than those treated with phosphatase, referred to as phosphorylation dependent, or $P(+)$ MAbs; and (3) those that preferentially recognized phosphatase-treated NF components, called dephosphorylation dependent, or $P(-)$ MAbs.

All NF-L-specific antibodies (categories VII and VIII), as well as MAbs recognizing core epitopes in NF-H and NF-M (categories III and VI), were of the $\mathrm{P}$ (ind) type. MAbs raised to native bovine NF proteins, and recognizing epitopes in the NF-H peripheral domain, were all of the $\mathrm{P}(+)$ type and represented, by far, the largest number of antibodies (categories I, IX, and X). $\mathrm{P}(-)$ type MAbs recognized only dephosphorylated NF-H within its peripheral domain and resulted from immunization with phosphatase-treated bovine NF proteins (see Lee et al., 1986). Antibodies monospecific for the NF-M peripheral domain were divided equally into $\mathrm{P}(+)$ and $\mathrm{P}($ ind) types (categories IV and V). Category IX antibodies, the largest group, were of the $\mathrm{P}(+)$ type with respect to peripheral domain epitopes in both NF-H and NF-M. No anti-bovine NF MAbs were yet obtained that 
Table 1. Reactivity of anti-bovine NF MAbs with other species

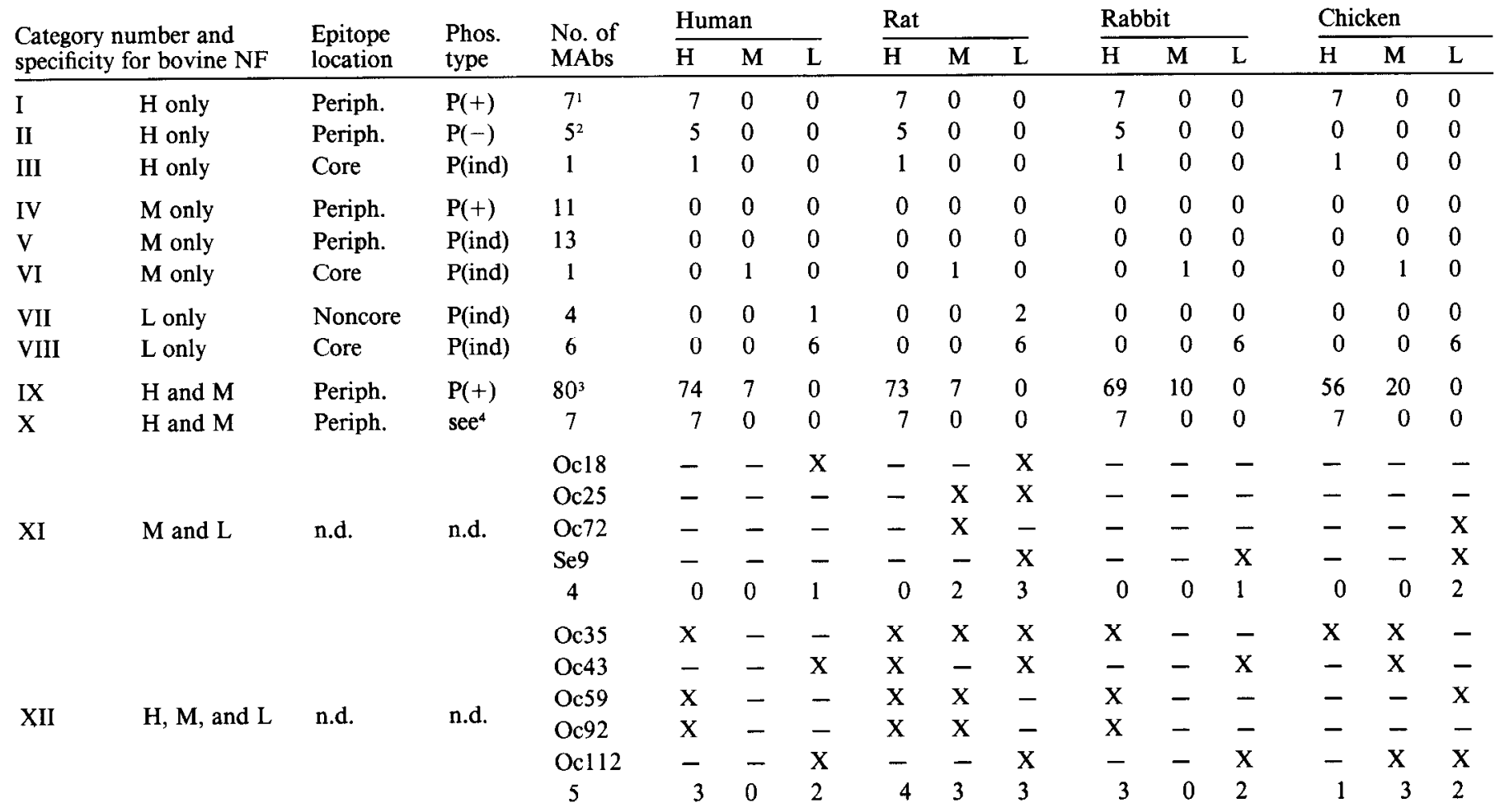

The 144 MAbs are divided into 12 categories (I-XII) on the basis of their specificity for bovine NF proteins, the approximate location of epitopes and the influence of antigen phosphorylation state on immunoreactivity (see Carden et al., 1985, and details in text). The numbers in each column under the headings $\mathrm{H}$, $\mathrm{M}$, or $\mathrm{L}$ indicate the number of MAbs in each category that immunoreacted positively with that NF polypeptide in each of the species indicated. The MAbs belonging to each of the categories (I-XII) have been identified individually in a previous report (Carden et al., 1985).

Notes: ${ }^{1}$ Immunoreactivity with NF-H was diminished on blots treated with phosphatase. ${ }^{2}$ Immunoreactivity with NF-H required pretreatment of blots with phosphatase. ${ }^{3}$ In all species, MAbs that immunoreacted with NF-M also detected NF-H. ${ }^{4}$ All MAbs were of the P(+)-type with respect to NF-H and of the P(ind)-type for NF-M. Abbreviations: Phos., phosphorylation; Periph., peripheral; n.d., not determined.

displayed $\mathrm{P}(\mathrm{ind})$ type immunoreactivity with the NF-H peripheral domain. Likewise, we have been unablc to produce MAbs that recognize dephosphorylated, or $\mathrm{P}(-)$ type, epitopes in the NF-M peripheral domain.

\section{Antibody specificity across species}

Characterization of the MAbs into 12 categories, as described above, allowed us to investigate whether 1 kind of epitope was more well-preserved than another, e.g., core versus peripheral, among NF components of other vertebrate species. The antibovine NF MAbs were therefore used for comparative immunoblotting of human (Fig. 4A), rat (Fig. $4 B$ ), rabbit (Fig. 4C), and chicken (Fig. 4D) NF proteins. Table 1 summarizes all of the information that was obtained. The immunoblots in Figure 4 show examples of MAbs that are monospecific for each NF component, and MAbs that cross-react between NF-H and NF-M in each species.

Antibodies in category I recognize bovine NF-H within their peripheral domain and in a phosphorylation-dependent, or $\mathrm{P}(+)$, manner. All retained immunoreactivity to, and specificity for, NF-H in the 4 other species. For each of the 7 MAbs in category I, treatment of immunoblots with phosphatase reduced NF-H immunoreactivity in all of the other species.

Category II MAbs also retained immunoreactivity with only NF-H in mammalian species, although it should be noted that exposure of blots to phosphatase was necessary in order to reveal NF-H immunoreactivity, since category II MAbs are of the $\mathrm{P}(-)$ type. Category II MAbs failed to recognize any chicken NF components, regardless of whether blots were treated with phos- phatase. Hence, these $\mathrm{P}(-)$ type peripheral domain epitopes are absent from chicken NF-H.

The core-specific MAb-, Ta56 (category III), recognized NF-H in all species tested and, furthermore, retained its monospecificity for this NF component.

The above results for NF-H-specific MAbs (categories I-III) suggest that $\mathrm{NF}-\mathrm{H}$ is well-preserved in mammals but differs slightly in the chicken. This observation contrasts dramatically with the situation found for NF-M (categories IV-V). All MAbs recognizing the peripheral domain of bovine NF-M were consistently negative during immunoblotting of NF-M from other species.

While the 24 MAbs to peripheral domain epitopes failed to recognize NF-M from non-bovine species, the sole NF-M corebinding $\mathrm{MAb}$ (category $\mathrm{VI}$ ) was consistently immunoreactive. Indeed, this explains why Ta54 was used in Figure 4 to immunostain NF-M in all non-bovine NF samples (see lane 3 in each of the panels, A-D).

Core-binding, NF-L-specific MAbs (category VIII) also crossreacted widely, recognizing this NF component in every other species. Noncore epitopes, on the other hand, were again much less well-preserved (see category VII).

The data described above concern MAbs monospecific for a single NF polypeptide. The largest group of MAbs (category IX) cross-reacted between NF-M and NF-H in bovine NF and was of the $\mathrm{P}(+)$ type with respect to each polypeptide. In other species, a large proportion of these MAbs became specific for NF-H alone by failing to recognize NF-M. This loss of immunoreactivity with NF-M parallels the behavior of category 


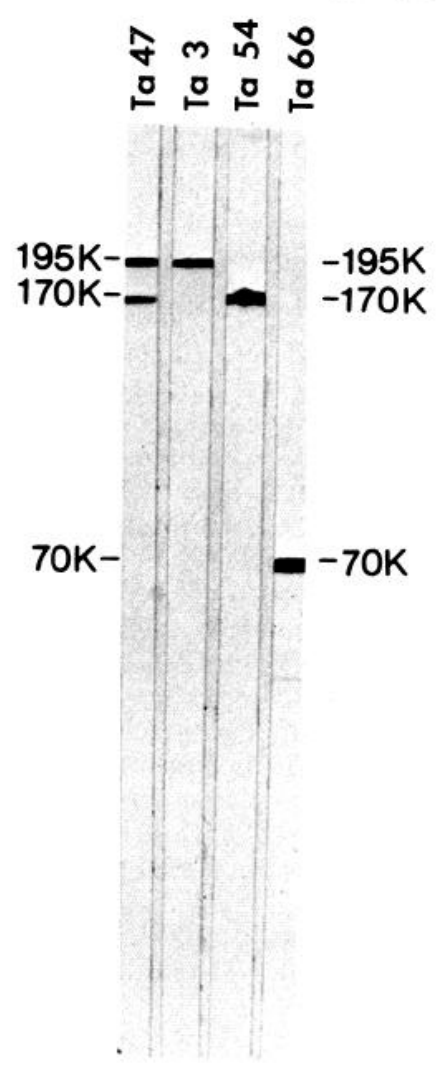

$\begin{array}{llll}1 & 2 & 3 & 4\end{array}$

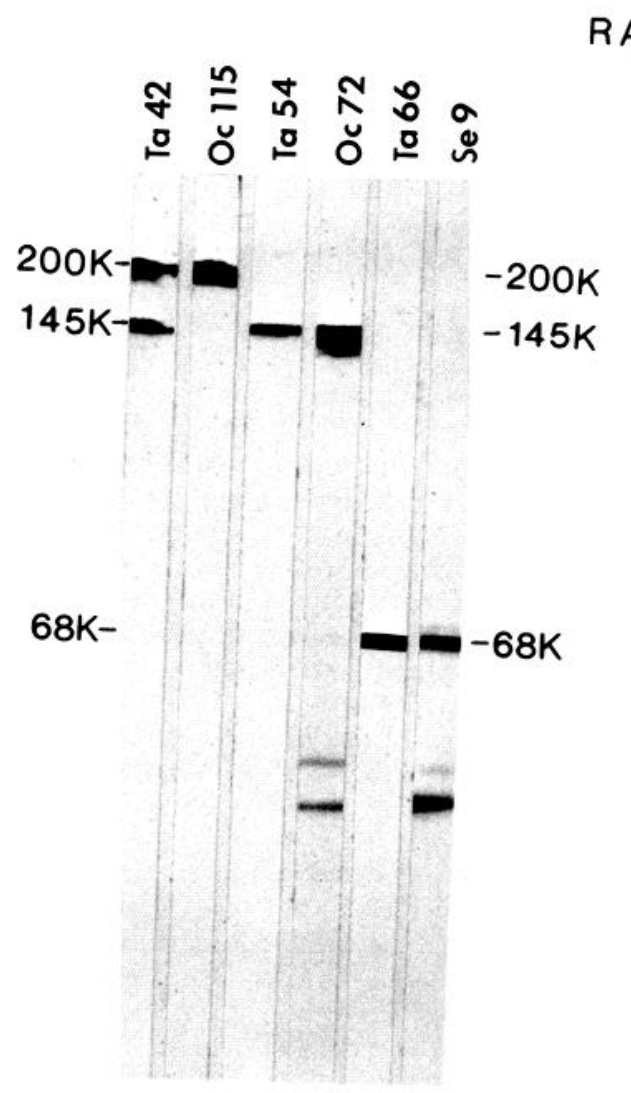

$\begin{array}{llllll}1 & 2 & 3 & 4 & 5 & 6\end{array}$

RABBIT

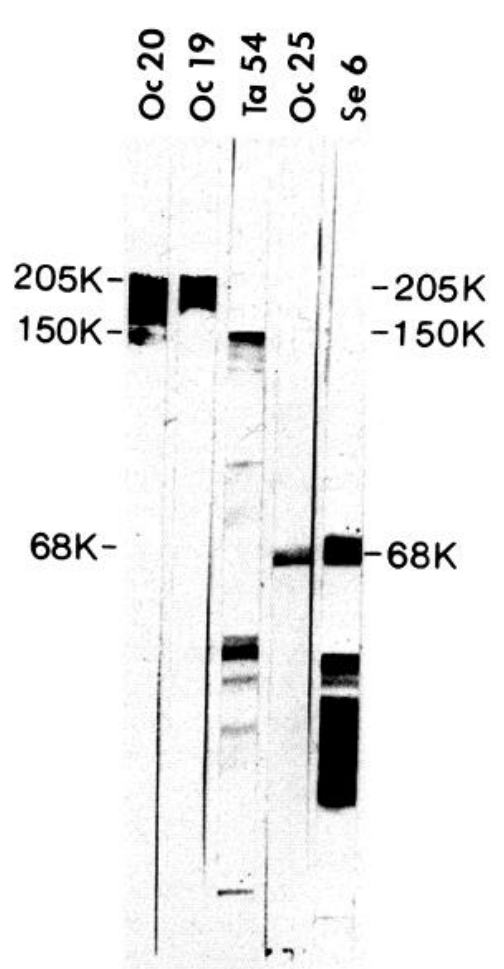

$\begin{array}{lllll}1 & 2 & 3 & 4 & 5\end{array}$

\section{CHICKEN}

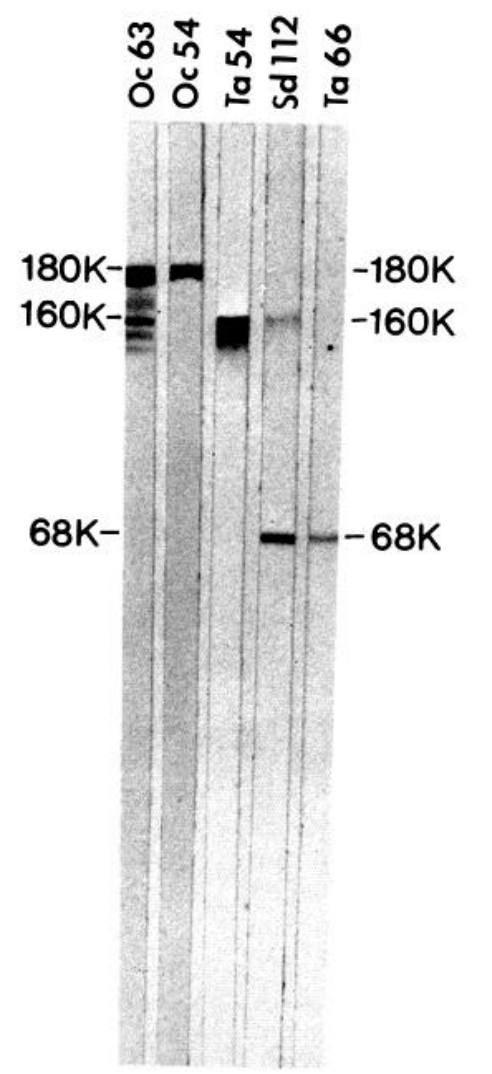

12345
Figure 4. Immunoblots of human, rat, rabbit, and chicken NF proteins. Nitrocellulose strips containing gelresolved human (A), rat (B), rabbit (C), or chicken (D) NF proteins were incubated with the MAbs indicated. Apparent molecular weights are indicated for NF-H, NF-M, and NF-L in each species. 
IV and V MAbs and provides further evidence to suggest that the peripheral domain of bovine NF-M has unique antigenic structure compared with the peripheral domains in other species. It should be noted, however, that around $15 \%$ of the MAbs in category IX retained immunoreactivity for NF-M in non-bovine species. For each of these MAbs, NF-H was always recognized in addition to NF-M, i.e., none became monospecific for NF-M from other species. Interestingly, chicken NF-M was detected by twice as many category IX MAbs as mammalian forms of NF-M.

Category X MAbs, like those in category IX, cross-reacted with epitopes on the peripheral domains of bovine NF-H and NF-M. However, their recognition of bovine NF-M was of a phosphorylation-independent, or P(ind), type. All of these MAbs lost immunoreactivity to NF-M, but retained immunoreactivity with NF-H, in all non-bovine species.

Cross-reacting MAbs in categories XI and XII showed no clear patterns as to their recognition of NF components from non-bovine species. For this reason, the data for each of the 9 MAbs in these categories are recorded in detail in Table 1.

\section{Discussion}

Previous studies on the cross-species immunoreactivity of NF polypeptides from higher vertebrates have affirmed the view that the heavy-, middle-, and light-molecular-weight ( $H, M$, and L) components of NF are distinct in any one species, but are immunologically related to the corresponding $\mathbf{H}, \mathbf{M}$, or L polypeptide of NF from other species (Shaw et al., 1984). Our results extend these studies by showing that the conservation of immunological cross-reactivity among NF polypeptides from different species is limited to MAbs recognizing epitopes located in particular domains of the NF polypeptides. Conversely, other domains in NF polypeptides appear to have undergone structural divergence, as evidenced by their diminished recognition when probed by antibodies that have been raised to, and react with, homologous domains of bovine NF polypeptides.

Antibodies recognizing core epitopes in each bovine NF polypeptide were immunoreactive with the corresponding polypeptide in all species tested. This suggests that some structural features of the core domains have been highly conserved during the evolution of NF polypeptides. Confirmation of this hypothesis, at least for NF-L, is provided by amino acid sequences of the mouse (Lewis and Cowan, 1985) and pig (Geisler et al., 1985 b), polypeptides in which core domains share more than $99 \%$ direct sequence homology. Since protein structure tends to be conserved in relation to functional activity (Dayhoff, 1972), it is likely that core domains are functionally important regions of the NF polypeptides. Furthermore, it should be noted that all IF polypeptides yet scquenced, including the 3 NF components, possess core domains whose consensus structural features are very similar (see Geisler et al., 1985b, and references therein). Thus, it is likely that core domains are instrumental in the formation of double-stranded coiled-coils that lead to the selfassembly of protofilaments and of $10 \mathrm{~nm}$ filaments, as suggested, for example, by Fuchs and Hanukoglu (1983), Crewther et al. (1984), and Weber and Geisler (1984). Similarities in the structural features of core domains among all IF polypeptides underlie the wide cross-reactivity of the anti-IFA MAb (Geisler et al., 1983; Pruss et al., 1981) and may explain the cross-reactivity between NF-L and vimentin by our core-binding MAb Se9.

While core domains from all IF polypeptides have similar secondary structural features, their amino acid sequences differ, especially among NF polypeptides. For example, comparison of core-domain sequences among porcine NF-H, NF-M, and NF-L reveals less than $50 \%$ direct homology (Geisler et al., 1985b). Similar values are obtained when NF polypeptides and non-neuronal IF components are compared (Geisler et al., $1985 b$ ). Structural variation among the core domains of NF polypeptides explains why we were able to obtain MAbs that recognized core epitopes unique to each of the bovine NF proteins. Since these epitopes are conserved across species, they may reflect a structure that is important in modulating interactions between different core domains during the assembly of filaments that are complex heteropolymers.

Only a few core-specific MAbs were obtained despite many fusion experiments, suggesting that NF core regions are poorly antigenic. Indeed, MAbs to bovine $\mathrm{NF}-\mathrm{L}$ were difficult to obtain, in line with the fact that more than $60 \%$ of the molecule forms that core domain (Geisler et al., 1985b). Paradoxically, animals immunized with NF-L for our fusion experiments developed moderate serum titers of anti-NF-L antibodies. MAbs to NF-H and NF-M were more easily obtained but, with rare exceptions, were directed to epitopes in their peripheral $\mathrm{COOH}$-terminal domains rather than to core epitopes. Geisler et al. (1985c) have shown that core domains remain associated as rigid, rodlike structures even after removal of their $\mathrm{NH}_{2}$ - and $\mathrm{COOH}$-terminal flanking sequences. The known tendency of rigid, poorly mobile protein domains to produce poor immunogenic responses (e.g., Robinson, 1984) might therefore explain the low yields of MAbs directed against NF core domains. Poor immunogenicity has been observed for the desmin core domain (Dahl et al., 1984). On the other hand, antisera and MAbs have been more readily obtained to core domain epitopes of GFAP (Dahl et al., 1984; Debus et al., 1983). It is our experience that MAbs specific to GFAP are more easily obtained than those to NF-L (Lee et al. 1984).

Complete cross-species immunoreactivity was also observed for category I MAbs, which recognize phosphorylation-dependent, or $\mathrm{P}(+)$, epitopes exclusive to the peripheral domain of NF-H. These findings demonstrate that the structural effects of phosphorylating NF-H have been tightly conserved in all species and indicate that NF-H phosphorylation is a universal phenomenon in higher vertebrates. More important, the conservation of phosphorylated epitopes has important functional implications, namely, that phosphorylation has an important role in the interaction of NF and surrounding axoplasmic constituents. Indeed, the observations that NF-II forms part of the side-arm projections between NF and microtubules (Hirokawa et al., 1984), together with our demonstrations that phosphorylation alters the structure of the NF-H peripheral domain (Carden et al., 1985), make it tempting to suggest that phosphorylation might regulatc sidc-arm function in all NF. It is of particular interest that the peripheral domain of NF-H interacts with microtubular proteins in vitro and that this interaction diminishes after NF dephosphorylation (Minami and Sakai, 1985).

Preservation of phosphorylated epitopes in the peripheral domain of NF-H was also apparent from the widespread crossspecies immunoreactivities of MAbs that cross-reacted with bovine NF-H and NF-M (categories IX and X). While all of these MAbs recognized epitopes in the phosphorylated form of NF-H, the avidity and the phosphate-dependency of their reactivities with NF-M were quite variable (Carden et al., 1985). Furthermore, these MAbs cross-reacted extensively with NF-H but only rarely with NF-M of other species, suggesting that some NF-H epitopes may have been duplicated in NF-M of bovine NF. Indeed, our findings complement those of Shaw et al. (1984), who observed that MAbs specific for NF-H of non-bovine species often cross-reacted with both NF-H and NF-M from bovine tissues and concluded that bovine NF-M differed from the homologous polypeptides in other species.

The peculiarity of bovine NF-M structure compared with that of NF-M from other species was further substantiated by the behavior of MAbs that were specific for bovine NF-M (categories IV and V). All of these MAbs failed to recognize non-bovine forms of NF-M, whether they reacted with phosphorylation-dependent (category IV) or phosphorylation-inde- 
pendent (category V) epitopes in the peripheral domain of NF-M. This profound lack of cross-species reactivity among epitopes in the peripheral domain of bovine NF-M contrasts with the preservation of immunological cross-reactivity of the core epitope (category VI) in the same polypeptide. Furthermore, the structural differences in the peripheral domain of bovine NF-M appear to be rather substantial, more than could be accounted for by the mere addition of NF-H elements. In addition, our data suggest that the peripheral domain of chicken NF-M also has some hybrid features, resembling bovine NF-M in sharing phosphorylated epitopes with NF-H (see category IX). Indeed, immunohistochemical studies have shown that chicken NF-M, like NF-H in other vertebrate species (Lee et al., 1986; Sternberger and Sternberger, 1983; Trojanowski et al., 1985), undergoes posttranslational phosphorylation during passage from perikaryon to axon (Bennett and DiLullo, 1985). Genetic translocations of NF-H and NF-M elements in bovine NF, and possibly in chicken NF as well, are supported by our data and should be looked for when sequence data become available.

Peripheral (noncore) domains of NF-L (category VII) were intermediate in the extent of cross-species preservation of epitopes, showing much greater variation than seen among the peripheral domains of NF-H but less than with NF-M. Again, core epilopes on NF-L (calegory VIII) were tightly conserved, in contrast to the peripheral portion of the same polypeptide. Comparative analysis of sequence data of NF-L from mouse (Lewis and Cowan, 1985) and pig (Geisler et al., 1985b) reveals variability in the COOH-terminal domains, in contrast to almost complete homology of core regions, corresponding to our immunological findings.

Finally, our survey of the immunological cross-reactivities among NF polypeptides of different species offers some useful information on the generation and use of antibody probes to NF polypeptides, especially when applied in differing species. By using bovine NF-M as an immunogen, for example, we have demonstrated a pitfall in the belief that antibodies generated to, and recognizing, particular NF polypeptides from one species will automatically be useful for demonstrating the same NF components in another species. Other probes, such as those that react with epitopes in core domains, are more difficult to obtain and often perform poorly by immunohistochemistry (Trojanowski et al., 1985), yet they retain cross-species immunoreactivities, thereby serving as potentially important tools in probing homologous components in different species. Most antisera to native NF proteins, on the other hand, are difficult to characterize biochemically because of their polyclonal nature and hence the multiplicity of epitopes that they recognize. Furthermore, the composition of these antisera probably reflects the high antigenicity of peripheral domains, and it is likely that their crossspecies immunoreactivities will rely on the extent to which they recognize phosphorylation-dependent epitopes in NF-H. Nevertheless, antibodies to peripheral domain epitopes are particularly useful because they generally perform well by immunohistochemistry (Trojanowski et al., 1985). It therefore seems apparent that a complete immunological mapping of NF polypeptides will require well-characterized probes that recognize unique and shared epitopes among the different domains. In addition, it may well be necessary that these probes be generated in response to immunization with NF components, or preferably defined fragments, that differ in phosphorylation state and are derived from the same species in which they are to be studied.

\section{References}

Bennett, G. S., and C. DiLullo (1985) Slow posttranslational modification of a neurofilament protein. J. Cell Biol. 100: 1799-1804.

Brown, B. A., R. A. Nixon, P. Strocchi, and C. A. Marotta (1981) Characterization and comparison of neurofilament proteins from rat and mouse CNS. J. Neurochem. 36: 143-153.
Calvert, R., and B. H. Anderton (1982) In vivo metabolism of mammalian neurofilament polypeptides in developing and adult rat brain. FEBS Lett. 145: 171-175.

Carden, M. J., W. W. Schlaepfer, and V. M-Y. Lee (1985) The structure, biochemical properties and immunogenicity of neurofilament peripheral regions are determined by phosphorylation. J. Biol. Chem. 260: 9805-9817.

Chin, T. K., P. A. M. Eagles, and A. Maggs (1983) The proteolytic digestion of ox neurofilaments with trypsin and $\alpha$-chymotrypsin. Biochem. J. 215: 239-252.

Chiu, F.-C., B. Korey, and W. T. Norton (1982) Intermediate filaments from bovine, rat and human CNS: Mapping analysis of the major proteins. J. Neurochem. 34: 1149-1 159.

Crewther, W. G., L. M. Dowling, P. M. Steinert, and D. A. D. Parry (1984) Structure of intermediate filaments. Int. J. Biol. Macromol. 5: $267-274$.

Czosnek, H., D. Soifer, and H. M. Wisniewski (1980) Studies on the biosynthesis of ncurofilament protcins. J. Cell Biol. 85: 726-734.

Dahl, D., D. C. Rueger, C. J. Crosby, and A. Bignami (1984) Cellspecific domains of glial- and muscle-type intermediate filament proteins. Exp. Cell Res. 154: 464-473.

Davison, P. F., and R. Jones (1980) Neurofilament proteins of mammals compared by peptide mapping. Brain Res. 182: 470-473.

Dayhoff, M. O. (1972) Atlas of Protein Sequence and Structure 1972, Vol. 5, National Biomedical Research Foundation, Silver Spring, MD.

Debus, E., K. Weber, and M. Osborn (1983) Monoclonal antibodies specific for glial fibrillary acidic (GFA) protein and for each of the neurofilament triplet polypeptides. Differentiation 25: 193-203.

Eagles, P. A. M., A. M. Maggs, and D. S. Gilbert (1980) Neurofilament structure and enzymic modification. Biochem. Soc. Trans. (Lond.) 8: 484-487.

Fuchs, E., and I. Hanukoglu (1983) Unravcling the structure of intermediate filaments. Cell 34: 332-334.

Geisler, N., S. Fischer, J. Vanderkerckhove, J. Van Damme, U. Plessmann, and K. Weber (1985a) Protein-chemical characterization of $\mathrm{NF}-\mathrm{H}$, the largest mammalian neurofilament component; intermediate filament-type sequences followed by a unique carboxy-terminal extension. EMBO J. 4: 57-63.

Geisler, N., E. Kaufmann, and K. Weber (1985b) Antiparallel orientation of the two double-stranded coiled coils in the tetrameric protofilament unit of intermediate filaments. J. Mol. Biol. 182: 173177.

Geisler, N., U. Plessmann, and K. Weber (1985c) The complete amino acid sequence of the major mammalian neurofilament protein (NF-L). FEBS Lett. 182: 475-478.

Geisler, N., E. Kaufmann, S. Fischer, U. Plessmann, and K. Weber (1983) Neurofilament architecture combines structural principles of intermediate filaments with carboxy-terminal extensions increasing in size between triplet proteins. EMBO J. 2: 1295-1302.

Hirokawa, N., M. A. Glicksman, and M. B. Willard (1984) Organization of mammalian neurofilament polypeptides within the neuronal cytoskeleton. J. Cell Biol. 98: 1523-1536.

Hogue-Angeletti, R., J. Q. Trojanowski, M. J. Carden, W. W. Schlaepfer, and V. M.-Y. Lee (1985) Domain structure of neurofilament subunits as revealed by monoclonal antibodies. J. Cell. Biochem. 27; 181-187.

Jones, S. M., and R. C. Williams (1982) Phosphate content of mammalian neurofilaments. J. Biol. Chem. 257: 9902-9905.

Julien, J.-P., and W. E. Mushynski (1982) Multiple phosphorylation sites in mammalian neurofilament polypeptides. J. Biol. Chem. 257: 10467-10470.

Julien, J.-P., and W. E. Mushynski (1983) The distribution of phosphyorlation sites among identified proteolytic fragments of mammalian neurofilaments. J. Biol. Chem. 258: 4019-4025.

Lazarides, E. (1982) Intermediate filaments. Annu. Rev. Biochem. 51: 219-245.

Lee, V, M.-Y., and C. D. Page (1984) The dynamics of nerve growth factor-induced neurofilament and vimentin filament expression and organization of PC12 cells. J. Neurosci. 4: 1705-1714.

Lee, V. M.-Y., H.-L. Wu, and W. W. Schlaepfer (1982) Monoclonal antibodies recognize individual neurofilament triplet proteins. Proc. Natl. Acad. Sci. USA 79: 6089-6092.

Lee, V. M.-Y., C. D. Page, H.-L. Wu, and W. W. Schlaepfer (1984) Monoclonal antibodies to gel excised glial filament protein and their 
reactivity with other intermediate filament proteins. J. Neurochem. 42: $25-32$.

Lee, V. M.-Y., M. J. Carden, and J. Q. Trojanowski (1986) Novel monoclonal antibodies provide evidence for the in situ existence of a nonphosphorylated form of the largest neurofilament subunit. J. Neurosci. 6: 850-858.

Lewis, S. A., and N. J. Cowan (1985) Genetics, evolution, and expression of the 68,000-mol-wt neurofilament protein: Isolation of a cloned cDNA probe. J. Cell Biol. 100: 843-850.

Liem, R. K. H., S. S. M. Chin, E. Moraru, and E. Wang (1985) Monoclonal antibodies to epitopes on different regions of the 200,000 dalton neurofilament protein. Exp. Cell Res. 156: 419-428.

Liem, R. K. H., S.-H. Yen, G. E. Solomon, and M. L. Shelanski (1978) Intermediate filaments in nervous tissues. J. Cell Biol. 79: 637-645.

Minami, Y., and H. Sakai (1985) Dephosphorylation suppresses the activity of neurofilament to promote tubulin polymerization. FEBS Lett. 185: 239-242.

Phillips, L. L., L. Autilio-Gambetti, and R. J. Lasek (1983) Bodian's silver method reveals molecular variation in the evolution of neurofilament proteins. Brain Res. 278: 219-223.

Pruss, R. M., R. Mirsky, M. Raff, R. Thorpe, A. J. Dowding, and B. H. Anderton (1981) All classes of intermediate filament share a common antigenic determinant defined by a monoclonal antibody. Cell 27: 419-428.

Robinson, A. L. (1984) Do antibodies prefer moving targets? Antibody recognition of their targets may be enhanced because they preferentially bind to the more mobile segments of protein antigens. Science 226: 819-822.

Schlaepfer, W. W., C. Lee, J. Q. Trojanowski, and V. M.-Y. Lee (1984) Persistence of immunoreactive neurofilament protein breakdown products in transected rat sciatic nerve. J. Neurochem. 43: 857-864. Shaw, G., E. Debus, and K. Weber (1984) The immunological relatedness of neurofilament proteins of higher vertebrates. Eur. J. Cell Biol. 34: 130-136.

Sternberger, L. A., and N. H. Sternberger (1983) Monoclonal antibodies distinguish phosphorylated and nonphosphorylated forms of neurofilaments in situ. Proc. Natl. Acad. Sci. USA 80: 6126-6130.

Trojanowski, J. Q., M. A. Obrocka, and V. M.-Y. Lee (1985) Distribution of bovine neurofilament subunits in neurons and neuronal processes: Immunohistochemical studies of bovine cerebellum with subunit specific monoclonal antibodies. J. Histochem. Cytochem. 33: $557-563$

Weber, K., and N. Geisler (1984) Intermediate filaments-From wool alpha-keratins to neurofilaments: A structural overview. In Cancer Cells, Vol. 1: The Transformed Phenotype, A. J. Levine, G. F. Vande Woude, W. C. Topp, and J. D. Watson, eds., pp. 153-159, Cold Spring Harbor Laboratory, New York.

Willard, M. B. (1976) Genetically determined protein polymorphism in the rabbit nervous system. Proc. Natl. Acad. Sci. USA 73: 36413645 . 